\title{
The Effect of Surgery on the Levels of Matrix Metalloproteinases in Patients with Inguinal Hernia
}

\author{
Sabina STROHALMOVÁ ${ }^{1,2}$, Kateřina LEVOVÁ ${ }^{2}$, Aleš Antonín KUBĚNA ${ }^{2}$, Zdeněk \\ KRŠKA ${ }^{1}$, David HOSKOVEC ${ }^{1}$, Tomáš ZIMA ${ }^{2}$, Marta KALOUSOVÁ ${ }^{2}$ \\ ${ }^{1} 1$ st Department of Surgery - Department of Abdominal, Thoracic Surgery and Traumatology, First \\ Faculty of Medicine, Charles University and General University Hospital in Prague, Prague, Czech \\ Republic, ${ }^{2}$ Institute of Medical Biochemistry and Laboratory Diagnostics, First Faculty of \\ Medicine, Charles University and General University Hospital in Prague, Prague, Czech Republic
}

Received December 1, 2020

Accepted April 13, 2021

Epub Ahead of Print June 2, 2021

\begin{abstract}
Summary
Matrix metalloproteinases (MMPs) are associated with the alteration of extracellular matrix. The purpose of this study was to investigate how the levels of matrix metalloproteinases and their inhibitors - TIMPs are influenced by the presence of inguinal hernia as well as by its surgical treatment. The studied group consisted of 25 patients with inguinal hernia and 21 healthy controls for comparison. Two blood samples - before and after the treatment were collected from patients. Serum concentrations of MMPs and TIMPs were analysed by multiplex immunoassays. There was a difference in circulating levels of MMPs in patients before the surgery compared to healthy controls - the concentrations of MMP-2 and MMP-9 were significantly lower $(p=0.026, p=0.018$, respectively). After the surgery, the levels of MMPs, especially MMP-2 $(p<0.0001)$, were significantly decreased in patients compared to the preoperative values, apart from MMP-9. On the contrary, MMP-9 showed significant increase after the surgery $(p<0.0001)$. Circulation levels of TIMP-2 in patients were significantly decreased in comparison with controls $(p=0.004)$, whereas levels of TIMP-1 were similar to controls. Both tested metalloproteinase inhibitors showed a significant decrease in detected levels (TIMP-1 $\mathrm{p}=0.0004$; TIMP-2 $\mathrm{p}<0.0001$ ) after the procedure compared to the preoperative values. The levels of MMPs, especially MMP-2 and MMP-9, and their inhibitors TIMP-1 and TIMP-2 are involved by the presence of inguinal hernia as well as are influenced by the surgery.
\end{abstract}

\section{Key words}

Matrix metalloproteinase $\bullet$ TIMP • Inguinal hernia

\section{Corresponding author}

Marta Kalousová, Institute of Medical Biochemistry and
Laboratory Diagnostics, First Faculty of Medicine, Charles University and General University Hospital in Prague, Kateřinská 32, 12108 Prague 2, Czech Republic. E-mail: marta.kalousova@lf1.cuni.cz

\section{Introduction}

Hernia is a pathological process, protrusion of tissues and organs outside their physiological position. There can be several types of hernias, such as umbilical, femoral, inguinal hernia, and the inguinal hernia is considered to be the most common type among the abdominal wall hernias (Dabbas et al. 2011, Ramanan et al. 2014, Zöller et al. 2013).

On the basis of the passage through the abdominal wall we can divide inguinal hernias to direct and indirect. Direct inguinal hernia protrudes through fossa inguinalis medialis to trigonum Hesselbachi and exits in anulus inguinalis superficialis. Indirect inguinal hernia is going through inguinal canal with spermatic cord in men or ligamentum teres uteri in women - it enters in fossa inguinalis lateralis (anulus inguinalis profundus) and exits in anulus inguinalis superficialis (Holzheimer 2005). Indirect inguinal hernia can be both congenital and acquired, direct inguinal hernia is only acquired. Risk factors of developing inguinal hernia are states or activities connected with higher intraabdominal pressure - for example excessive effort, cough, constipation (Jiang et al. 2015). The treatment of inguinal hernia is surgical, while elective surgery is preferred. 
Matrix metalloproteinases (MMPs) are a family of endopeptidases which contribute to various physiological and pathological processes. They degrade connective tissue and therefore participate in processes related to ontogenetical development, such as morphogenesis, angiogenesis, growth or wound healing (Cauwe et al. 2007, Lee et al. 2004). MMPs play a role in all the processes connected with increased synthesis, degradation, organisation or maturation of extracellular matrix. Thus, they are key regulators of numerous aspects of tissue remodeling and repair (Gill et al. 2008). Furthermore, altered activity of MMPs is linked with degenerative processes, as well as affects inflammatory response or cancer development (Gill et al. 2008, Jabłońska-Trypuć et al. 2016, Rosenberg 2009, Roy et al. 2009).

It has been previously reported that altered activity of metalloproteinases affects the etiopathogenesis of inguinal hernia through the imbalance in the rate of collagen metabolism (Antoniou et al. 2009, Henriksen et al. 2015, Klinge et al. 1999, Peeters et al. 2014).

MMPs action is mainly influenced by their endogenous tissue inhibitory proteins (TIMPs). Hence, the offset between TIMPs and MMPs levels is important for the extracellular matrix remodelling in the tissue.

The purpose of the study was to investigate how the circulating levels of matrix metalloproteinases and their inhibitors - TIMPs are influenced by the presence of inguinal hernia as well as by its surgical treatment.

\section{Methods}

\section{Study objects}

The study group consisted of 25 patients diagnosed with inguinal hernia (22 men and 3 women, median age 72, IQR 55-79 years) from the $1^{\text {st }}$ Department of Surgery - Department of Abdominal, Thoracic Surgery and Traumatology, First Faculty of Medicine, Charles University and General University Hospital in Prague. These patients underwent elective surgery and had no signs of incarcerated hernia. Patients were cardiopulmonary compensated and without symptoms of any infection. Patients with malignancies were not included in the study.

As for the type of hernia, eight patients had direct inguinal hernia, ten patients indirect hernia, three patients had combination of direct and indirect hernia. Four patients suffered from recidive of inguinal hernia. Fourteen patients were treated for arterial hypertension, ten patients with mild hypertension, four with severe hypertension. One of the patients with severe hypertension suffered from hypertensive kidney disease (serum level of creatinine $140 \mu \mathrm{mol} / \mathrm{l}$ ). Two patients were treated for diabetes mellitus on the treatment with GLP1 - agonists, one with complications - diabetic neuropathy and diabetic nephropathy (serum level of creatinine below $150 \mu \mathrm{mol} / \mathrm{l})$. Another patient had chronic kidney disease, creatinine $109 \mu \mathrm{mol} / \mathrm{l}$. Eleven patients were diagnosed with heart diseases - five patients with ischemic heart disease, seven patients with atrial fibrillation, one patient with aortic regurgitation. Six patients had no internal comorbidities.

Twenty one healthy controls (eight men and thirteen women, median age 41 (IQR 30-53 years)) were used for comparison baseline levels before the surgery. Controls were volunteers without presence of inguinal hernia, without symptoms of acute infection or disease, without elevation of inflammatory markers in blood (CRP, leucocytes), without hepatic or renal failure, without presence of a tumour, and with blood count in the reference range. They were not taking any chronic medication. Healthy volunteers without internal comorbidities or presence of hernia were chosen to avoid the influence on the levels of MMPs. Results of routine laboratory examinations of the studied groups are presented in Table 1.

This study was performed in adherence to the principles of the Helsinki Declaration and approved by the Ethics Committee of The General University Hospital in Prague. All subjects gave their informed consent with participation in the study.

\section{Laboratory analyses}

Blood samples from all individuals were obtained after overnight fasting from the cubital vein into tubes without anticoagulant to obtain serum. From all patients, blood collection occured preoperatively 1-3 $\mathrm{h}$ before the surgery and postoperatively 24 hours after the surgery. Blood collection occurred together with blood collection for standard laboratory analyses. Test tubes with blood were after coagulation centrifuged for $15 \mathrm{~min}$ at $900 \mathrm{x} \mathrm{g}$ and serum was stored at $-80{ }^{\circ} \mathrm{C}$ until measurement of metalloproteinases and selected inhibitors.

\section{Multiplex xMAP assay}

Serum samples were analyzed with a 5 matrix metalloproteinases MILLIPLEX MAP Human MMP Magnetic Bead Panel 2 kit for simultaneous analysis of 
Table 1. Basic laboratory characteristics of the study groups

\begin{tabular}{lccc}
\hline Parameter & Patients & Healthy Controls & p value \\
\hline Number & 25 & 21 & $<0.0001$ \\
Age $($ years $)$ & $72(55-79)$ & $41(30-53)$ & $\mathrm{ns}$ \\
Protein $(\mathrm{g} / \mathrm{l})$ & $69(66-71)$ & $72(67-74)$ & $\mathrm{ns}$ \\
CRP $(\mathrm{mg} / \mathrm{l})$ & $1.8(1.0-4.1)$ & $3.1(1.5-4.3)$ & $\mathrm{ns}$ \\
ALT $(\mu \mathrm{kat} / \mathrm{l})$ & $0.34(0.28-0.49)$ & $0.40(0.31-0.48)$ & $\mathrm{ns}$ \\
AST $(\mu \mathrm{kat} / \mathrm{l})$ & $0.42(0.36-0.48)$ & $0.37(0.30-0.45)$ & $\mathrm{ns}$ \\
GGT $(\mu \mathrm{kat} / \mathrm{l})$ & $0.43(0.3-1.0)$ & $0.36(0.30-0.56)$ & $\mathrm{ns}$ \\
Urea $(\mathrm{mmol} / \mathrm{l})$ & $6.2(4.9-7.2)$ & $4.9(3.9-6.4)$ & $\mathrm{ns}$ \\
Creatinine $(\mu \mathrm{mol} / \mathrm{l})$ & $84(78-97)$ & $65(58-77)$ & 0.026 \\
Hemoglobin $(\mathrm{g} / \mathrm{l})$ & $147(142-154)$ & $6.5(5.7-8.4)$ & $\mathrm{ns}$ \\
Leukocytes $\left(x 10^{9} / \mathrm{l}\right)$ & $6.5(4.8-8.2)$ & $243(204-305)$ & $\mathrm{ns}$ \\
Thrombocytes $\left(x 10^{9} / \mathrm{l}\right)$ & $214(178-252)$ & & \\
\hline
\end{tabular}

All data shown as median (interquartile range). Abbreviations: CRP - C-reactive protein, ALT - Alanine aminotransferase, AST Aspartate aminotransferase, GGT - Gamma-glutamyltransferase. All results were considered statistically significant at $p<0.05$, ns, not significant.

tested metalloproteinases (MMP-1,-2,-7,-9,-10) and with MILLIPLEX MAP Human TIMP Magnetic Bead Panel 1 kit (Merck KGaA, Darmstadt, Germany) to obtain the concentrations of TIMP-1 and TIMP-2 inhibitors. Analytes were quantified using MAGPIX system, which utilizes XMAP technology and data acquisition xPONENT software (Luminex Corp., Austin, TX, USA). Data were then analyzed by MILLIPLEX ${ }^{\circledR}$ Analyst 5.1 software (Merck KGaA, Darmstadt, Germany). Concentrations of analytes were determined using 5PL curve fit. Two quality controls (low, high) were run with each assay. All quality control results were found to fall within the expected ranges provided by the manufacturer. Results of all MMPs and TIMPs are expressed in $\mathrm{ng} / \mathrm{ml}$.

\section{Statistical analysis}

Statistical tests were used to examine the differences among studied groups (patients before and after treatment, controls). We used the nonparametric Mann Whitney U test for group comparison (age), and the Generalized Linear Model with Gamma Distribution and reciprocal link function for comparisons with adjustment for sex and age (biochemical variables). Differences between patient's serum analytes levels before and after the treatment were analyzed using Wilcoxon signed rank test. Spearman's rank correlation coefficient was used to determine the correlations between studied analytes. Data are expressed as median (IQR, interquartile range). Differences among the subgroups with different types of hernia were evaluated using the Kruskal-Wallis test. All results were considered statistically significant at $\mathrm{p}<0.05$ and all reported $\mathrm{p}$ values were 2-sided. Statistical analysis was performed using GraphPad Prism software 8 (San Diego, CA, USA) and for Generalized linear model the Wolfram Mathematica software, version 11.3 (United Kingdom) was used.

\section{Results}

Serum values of tested metalloproteinases and selected main inhibitors were analyzed at two stages before and after the surgery, and the corresponding analytes' levels were additionally compared to the healthy control group.

Serum concentrations of metalloproteinases and TIMPs in patients compared to the control group are listed in Table 2. Patients with inguinal hernia had significantly decreased preoperative concentrations of MMP-2 and MMP-9 ( $\mathrm{p}=0.026, \mathrm{p}=0.018$, respectively), whereas circulating blood levels of MMP-1,-7,-10 were without change. Different trends were likewise found for tested inhibitors. Preoperative levels of TIMP-2 in patients were significantly decreased in comparison with the control group ( $p=0.004)$, whereas levels of TIMP-1 were similar to controls.

Patients' serum levels of all tested analytes were significantly different when compared blood draw before and after the surgery (Table 2). After surgery there was a significant decrease in MMPs levels apart from MMP-9, while most significant decrease was observed 
Table 2. MMPs and TIMPs serum levels of patients and control group.

\section{Patients}

\begin{tabular}{|c|c|c|c|c|c|}
\hline $\begin{array}{l}\text { Analyte } \\
\text { ng/ml }\end{array}$ & Before & After & Controls & $\begin{array}{c}\text { p value } \\
\text { Before vs After }\end{array}$ & $\begin{array}{c}\text { p value } \\
\text { Before vs controls }\end{array}$ \\
\hline$M M P-1$ & $\begin{array}{c}9.5 \\
(7.0-12.2)\end{array}$ & $\begin{array}{c}9.2 \\
(6.6-12.0)\end{array}$ & $\begin{array}{c}5.5 \\
(3.3-10.7)\end{array}$ & 0.016 & ns \\
\hline$M M P-2$ & $\begin{array}{c}112.3 \\
(92.9-126.0)\end{array}$ & $\begin{array}{c}91.4 \\
(80.9-103.8)\end{array}$ & $\begin{array}{c}117.4 \\
(100.7-131.9)\end{array}$ & $<0.0001$ & 0.026 \\
\hline$M M P-7$ & $\begin{array}{c}13.8 \\
(10.7-19.6)\end{array}$ & $\begin{array}{c}12.5 \\
(9.7-16.3)\end{array}$ & $\begin{array}{c}11.6 \\
(8.7-12.9)\end{array}$ & 0.021 & ns \\
\hline$M M P-9$ & $\begin{array}{c}133.5 \\
(99.3-210.9)\end{array}$ & $\begin{array}{c}345.3 \\
(254.5-455.4)\end{array}$ & $\begin{array}{c}236.4 \\
(177.5-279.0)\end{array}$ & $<0.0001$ & 0.018 \\
\hline$M M P-10$ & $\begin{array}{c}0.44 \\
(0.33-0.72)\end{array}$ & $\begin{array}{c}0.44 \\
(0.29-0.54)\end{array}$ & $\begin{array}{c}0.44 \\
(0.35-0.54)\end{array}$ & 0.014 & ns \\
\hline TIMP-1 & $\begin{array}{c}103.8 \\
(93.9-115.4)\end{array}$ & $\begin{array}{c}90.9 \\
(79.1-109.2)\end{array}$ & $\begin{array}{c}100.4 \\
(91.7-112.5)\end{array}$ & 0.0004 & ns \\
\hline TIMP-2 & $\begin{array}{c}45.8 \\
(42.4-47.8)\end{array}$ & $\begin{array}{c}39.5 \\
(37.0-41.7)\end{array}$ & $\begin{array}{c}48.2 \\
(44.3-52.3)\end{array}$ & $<0.0001$ & 0.004 \\
\hline
\end{tabular}

Data are expressed as median (interquartile range). All results were considered statistically significant at $p<0.05$, ns, not significant.

A

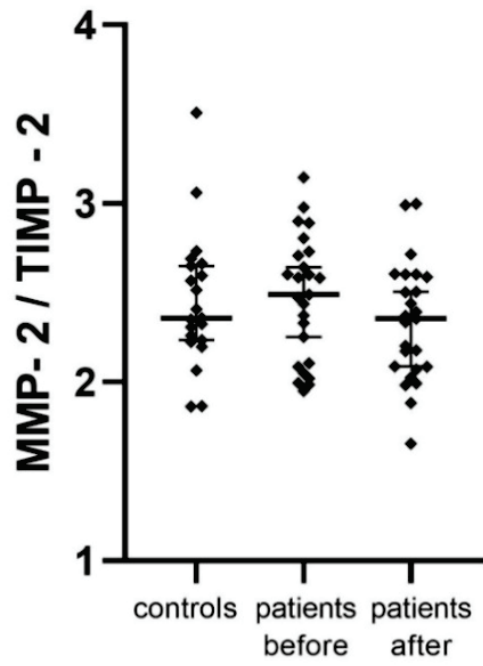

B

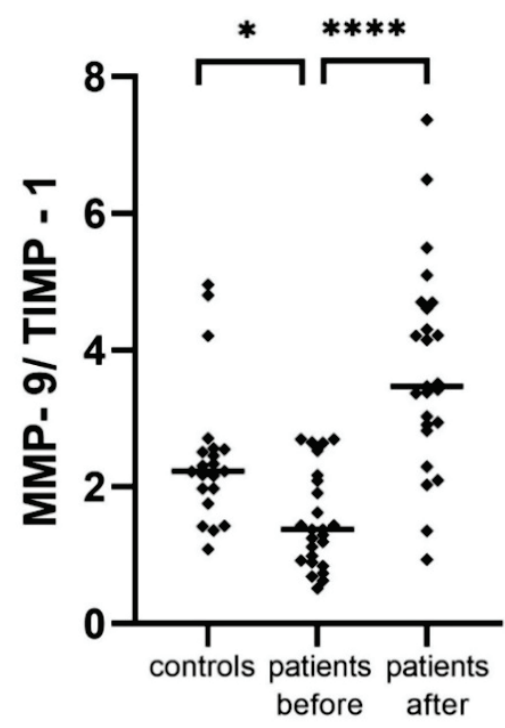

Fig. 1. Ratios of MMPs/TIMPs among the study groups, $* p=0.026 ; * * * * p<0.0001$

for MMP-2 $(\mathrm{p}<0.0001)$. In contrast, we observed a significant elevation of MMP-9 levels $(p<0.0001)$ after the surgery. Both tested metalloproteinase inhibitors showed a significant decrease in detected levels (TIMP-1 $\mathrm{p}=0.0004$; TIMP-2 $\mathrm{p}<0.0001$ ) after the procedure compared to the preoperative values. We did not observe any differences in patients MMPs/TIMPs levels when evaluating subgroups with various types of hernia (direct or indirect, combination of direct/indirect or recidive).
The correlation between tested analytes was also determined. There was a significant correlation between MMP-2 and TIMP-2 in both patients before the surgery and controls $(\rho=0.66, p=0.0004 ; \rho=0.64, p=0.002$, respectively) and this correlation was no longer apparent after the procedure. However, after the surgery both inhibitors TIMP-1,2 were significantly correlated $(\rho=0.64, p=0.001)$.

Serum levels of MMP-2 and MMP-9 were also 
evaluated as ratios with corresponding dominant inhibitors (Fig. 1). The MMP-9/TIMP-1 preoperative levels ratio was significantly decreased compared to the control group $(\mathrm{p}=0.026)$, and after the procedure there was a significant increase with respect to the preoperative levels $(p<0.0001)$, thereby MMP-9/TIMP-1 ratio copied the same trend as MMP-9 serum levels.

\section{Discussion}

In this study, we analyzed the circulating levels of MMPs and TIMPs in patients with inguinal hernia before and after the surgical treatment and compared the preoperative values to healthy controls.

Inguinal hernia formation is connected with tissue alteration. The process of hernia development is associated with the disturbance of regulation of metabolism of collagen (Henriksen et al. 2011). MMPs enzymes play a key role in extracellular matrix metabolism during tissue remodeling and repair (Gill et al. 2008). They are predominantly extracellularly, however several studies have shown that MMP-1 (Limb et al. 2005), MMP-2 (Aldonyte et al. 2009, Kwan et al. 2004), MMP-11 (Luo et al. 2002) and MMP-13 (Cuadrado et al. 2009) can also be found intracellularly and may act on intracellular proteins.

In our study, we focused on how are the circulating levels of tested analytes influenced by the presence of inguinal hernia as well as by the surgery. We found significant differences in the preoperative levels of MMP-2, MMP-9 and TIMP-2 compared to the controls. While preoperative serum levels of MMP-2, MMP-9 and TIMP-2 were decreased $(\mathrm{p}=0.026, \mathrm{p}=0.019, \mathrm{p}=0.004$, respectively), levels of MMP-1, MMP-10 and TIMP-1 were similar to controls.

There are several studies, which were also concentrated on the circulating levels of matrix metalloproteinases and their inhibitors in inguinal hernias. But the levels of MMPs both in serum and plasma, especially for MMP-2 and MMP-9, as well as for TIMPs were reported inconsistently.

Relationship between inguinal hernia and selected preoperative serum levels of MMPs as well as TIMPs in patients with direct, indirect and bilateral type of hernia was investigated by Isik and coworkers. When compared to healthy donors, all three groups of patients revealed significant elevation of all four tested MMPs (MMP-1,2,9,13) whereas levels of TIMPs were decreased. These analytes were also tested in tissue homogenates taken from transversalis fascia with similar trend results as for serum samples (Isik et al. 2017). In contrast, our data indicate that serum preoperative levels of MMP-1 and TIMP-1 were without significant changes and MMP-2,-9 were significantly decreased compared to the controls $(\mathrm{p}=0.026, \mathrm{p}=0.018$, respectively). Significant decrease of TIMP-2 was in line with our results.

Decreased circulating levels of MMP-2,-9 were previously also reported in plasma of patients with inguinal hernia (Antoniou G. A. et al. 2011). In this study, 91 patients and 35 controls were compared for both circulating and tissue levels of MMP-2,9 and TIMP-1,2. Whereas their results of tested biomarkers in tissue of the abdominal fascia were consistent with those reported by Isik et al. (2017), they differed in circulating preoperative levels. Antoniou et al. (2011) found plasma concentrations of MMP-9 and MMP-2 significantly decreased in patients with inguinal hernia in comparison with the control group, which corresponds to our results. On the other hand, several former studies reported significantly higher MMP-2 levels in both serum and tissue samples in patients with direct or indirect inguinal hernia than those in controls (Bellón et al. 2001, Li et al. 2018, Smigielski et al. 2011, Smigielski et al. 2009, Wang et al. 2020).

Serum MMP-2,-9 were also tested in patients with inguinal hernias along with procollagen type I,III N-terminal propeptides (PINP, PIIINP, respectively) (Li et al. 2018). In contrast to our MMP-9 results, as well as Isik et al. (2017) and Antoniou et al. (2011), they observed no significant changes in serum MMP-9 preoperative levels between the patients and controls (Li et al. 2018).

MMPs proteolytic activities influence crucial cellular processes as well as many other physiological events and their activities are mainly regulated by TIMPs. Results regarding the circulating plasma levels of TIMP-1,-2 were also previously reported differently. Significantly higher circulating plasma levels in patients with inguinal hernia compared to controls were previously reported for TIMP-2 and decresed levels of TIMP-1 (Antoniou et al. 2011). Furthermore, elevation of TIMP-2, but only in recurrent inguinal hernia patients were noticed by Smigielski et al. (2011), while the levels of TIMP-2 in other groups (direct, indirect hernias) were similar to controls. On the other hand significantly decreased serum levels of TIMP-1,2,3 compared to control group were reported (Isik et al. 2017). In our study preoperative levels of TIMP-2 were significantly 
decreased in comparison with controls $(p=0.004)$, whereas levels of TIMP-1 were similar to controls. Thus, the decrease of circulating levels of MMP-9 in patients with inguinal hernia before the surgery was not followed by an increase of the main MMP-9 inhibitor - TIMP-1, because TIMP-1 serum levels were similar to those detected in controls. We found a significant correlation between both inhibitors (TIMP-1, TIMP-2) $(\rho=0.64$, $\mathrm{p}=0.001$ ) after the surgery, where both inhibitors showed a significant decrease in detected levels (TIMP-1 $\mathrm{p}=0.0004$; TIMP-2 $\mathrm{p}<0.0001$ ) compared to preoperative values.

Some previous studies were focused on differences in MMPs levels according to the type of hernia. We did not observe any differences in patients MMPs and TIMPs levels both before and after the procedure when they were evaluated according to the type of hernia (direct or indirect). These results are in agreement with those previously reported for MMP-1,-2,-9 (Isik et al. 2017, Li et al. 2018, Smigielski et al. 2009).

By reason of each TIMP displays preferential MMP-binding specificity, the MMP/TIMP ratios can determine the overall proteolytic activity. We observed a higher MMP-9/TIMP-1 ratio of circulating levels in patients after the procedure compared to the preoperative values, consistent with a net MMP-9 increase in patients after surgery. Significant elevation of MMP-2/TIMP-2 mRNA ratios in primary inguinal hernias patients (both direct and indirect) compared to the controls was previously reported, where the MMP-2 and TIMP-2 expresion in anterior rectus sheat fascia was done (Wang et al. 2020). In contrast, we did not observed a significant change in MMP-2/TIMP-2 circulating levels ratio between patients (blood draw before and after the surgery) and control group.

Most studies concerned with MMPs and TIMPs circulating levels at a single time point - as preoperative values. Our study was aimed to describe the changes in biomarkers levels before and after the surgical procedure. In patients after surgery, there was a significant decrease in MMPs levels, except for MMP-9. MMP-9 showed a significant increase $(\mathrm{p}<0.0001)$. Within the standardization of procedures, we dealt with the impact of surgical intervention on selected biomarkers. By default, samples are taken into the biobank prior to the planned surgical intervention and any subsequent sampling can significantly affect the results. The results of the study confirm that the sampling time with respect to the surgical performance can significantly affect the levels of the studied parameters. Standardization of the collection time into the biobank with respect to the surgical performance is therefore a very important element for ensuring the comparability of the collected samples.

We have to admit some limitations of our study. Control group individuals were notably younger than patients with inguinal hernia. Furthermore, the percentage of women in the control group was higher than in the patient's group. Comparisons of MMPs and TIMPs levels between the groups were therefore statistically adjusted for the age and sex differences. Likewise, statistical evaluation of differences in MMPs and TIMPs levels according to the type of hernia has an informative value only between groups with direct and indirect type of hernia, due to the small number of patients in two remaining groups - combination of direct/indirect and recidive inguinal hernia.

The levels of MMPs, especially MMP-2 and MMP-9, and their inhibitors TIMP-1 and TIMP-2 are involved by the presence of inguinal hernia as well as are influenced by the surgery.

\section{Conflict of Interest}

There is no conflict of interest.

\section{Acknowledgements}

This work was supported by European Regional Development Fund project [EF16_013/0001674], by Ministry of Health, Czech Republic - conceptual development of research organization [VFN-64165], and by research projects Progres Q25, and SVV [260524/2020]. The authors are thankfull to the laboratory staff mainly to Mrs. Dita Hudcová and Mrs. Marcela Bartáková from the Institute of Medical Biochemistry and Laboratory Diagnostics, First Faculty of Medicine, Charles University and General University Hospital in Prague for technical assistance. The authors would like to thank also to physicians and nurses from the First Department of Surgery - Department of Abdominal, Thoracic Surgery and Traumatology, First Faculty of Medicine, Charles University and General University Hospital in Prague. 


\section{References}

ALDONYTE R, BRANTLY M, BLOCK E, PATEL J, ZHANG J: Nuclear localization of active matrix metalloproteinase-2 in cigarette smoke-exposed apoptotic endothelial cells. Exp Lung Res 35: 59-75, 2009. https://doi.org/10.1080/01902140802406059

ANTONIOU GA, TENTES IK, ANTONIOU SA, SIMOPOULOS C, LAZARIDES MK: Matrix metalloproteinase imbalance in inguinal hernia formation. J Invest Surg 24: 145-150, 2011. https://doi.org/10.3109/08941939.2011.558610

ANTONIOU SA, ANTONIOU GA, GRANDERATH FA, SIMOPOULOS C: The role of matrix metalloproteinases in the pathogenesis of abdominal wall hernias. Eur J Clin Invest 39: 953-959, 2009. https://doi.org/10.1111/j.1365-2362.2009.02199.x

BELLÓN JM, BAJO A, GA-HONDUVILLA N, GIMENO MJ, PASCUAL G, GUERRERO A, BUJÁN J: Fibroblasts from the transversalis fascia of young patients with direct inguinal hernias show constitutive MMP-2 overexpression. Ann Surg 233: 287-291, 2001. https://doi.org/10.1097/00000658-200102000-00020

CAUWE B, VAN DEN STEEN PE, OPDENAKKER G: The biochemical, biological, and pathological kaleidoscope of cell surface substrates processed by matrix metalloproteinases. Crit Rev Biochem Mol Biol 42: 113-185, 2007. https://doi.org/10.1080/10409230701340019

CUADRADO E, ROSELL A, BORRELL-PAGES M, GARCIA-BONILLA L, HERNANDEZ-GUILLAMON M, ORTEGA-AZNAR A, MONTANER J: Matrix metalloproteinase-13 is activated and is found in the nucleus of neural cells after cerebral ischemia. J Cereb Blood Flow Metab 29: 398-410, 2009. https://doi.org/10.1038/jcbfm.2008.130

DABBAS N, ADAMS K, PEARSON K, ROYLE G: Frequency of abdominal wall hernias: is classical teaching out of date? JRSM Short Reports 2: 5, 2011. https://doi.org/10.1258/shorts.2010.010071

GILL SE and PARKS WC: Metalloproteinases and their inhibitors: regulators of wound healing. Int J Biochem Cell Biol 40: 1334-1347, 2008. https://doi.org/10.1016/j.biocel.2007.10.024

HENRIKSEN NA, MORTENSEN JH, SORENSEN LT, BAY-JENSEN AC, AGREN MS, JORGENSEN LN, KARSDAL MA: The collagen turnover profile is altered in patients with inguinal and incisional hernia. Surgery 157: 312-321, 2015. https://doi.org/10.1016/j.surg.2014.09.006

HENRIKSEN NA, YADETE DH, SORENSEN LT, AGREN MS, JORGENSEN LN: Connective tissue alteration in abdominal wall hernia. Br J Surg 98: 210-219, 2011. https://doi.org/10.1002/bjs.7339

HOLZHEIMER RG: Inguinal Hernia: classification, diagnosis and treatment--classic, traumatic and Sportsman's hernia. Eur J Med Res 10: 121-134, 2005.

ISIK A, GURSUL C, PEKER K, AYDIN M, FIRAT D, YILMAZ I: Metalloproteinases and their inhibitors in patients with inguinal hernia. World J Surg 41: 1259-1266, 2017. https://doi.org/10.1007/s00268-016-3858-6

JABŁOŃSKA-TRYPUĆ A, MATEJCZYK M, ROSOCHACKI S: Matrix metalloproteinases (MMPs), the main extracellular matrix (ECM) enzymes in collagen degradation, as a target for anticancer drugs. J Enz Inhib Med Chem 31: 177-183, 2016. https://doi.org/10.3109/14756366.2016.1161620

JIANG ZP, YANG B, WEN LQ, ZHANG YC, LAI DM, LI YR, CHEN S: The etiology of indirect inguinal hernia in adults: congenital or acquired? Hernia 19: 697-701, 2015. https://doi.org/10.1007/s10029-014-1326-5

KLINGE U, ZHENG H, SI Z, SCHUMPELICK V, BHARDWAJ RS, MUYS L, KLOSTERHALFEN B: Expression of the extracellular matrix proteins collagen I, collagen III and fibronectin and matrix metalloproteinase-1 and -13 in the skin of patients with inguinal hernia. Eur Surg Res 31: 480-490, 1999. https://doi.org/10.1159/000008728

KWAN JA, SCHULZE CJ, WANG W, LEON H, SARIAHMETOGLU M, SUNG M, SAWICKA J, SIMS DE, SAWICKI G, SCHULZ R: Matrix metalloproteinase-2 (MMP-2) is present in the nucleus of cardiac myocytes and is capable of cleaving poly (ADP-ribose) polymerase (PARP) in vitro. FASEB J 18: 690-692, 2004. https://doi.org/10.1096/fj.02-1202fje

LEE MH, MURPHY G: Matrix metalloproteinases at a glance. J Cell Sci 117: 4015-4016, 2004. https://doi.org/10.1242/jcs.01223 
LI J, ZHANG X, SUN Q, LI W, YU A, FU H, CHEN K: Circulating matrix metalloproteinases and procollagen propeptides in inguinal hernia. Hernia 22: 541-547, 2018. https://doi.org/10.1007/s10029-018-1751-y

LIMB GA, MATTER K, MURPHY G, CAMBREY AD, BISHOP PN, MORRIS GE, KHAW PT: Matrix metalloproteinase-1 associates with intracellular organelles and confers resistance to lamin $\mathrm{A} / \mathrm{C}$ degradation during apoptosis. Am J Pathol 166: 1555-1563, 2005. https://doi.org/10.1016/S0002-9440(10)62371-1

LUO D, MARI B, STOLL I, ANGLARD P: Alternative splicing and promoter usage generates an intracellular stromelysin 3 isoform directly translated as an active matrix metalloproteinase. J Biol Chem 277: 25527-25536, 2002. https://doi.org/10.1074/jbc.M202494200

PEETERS E, DE HERTOGH G, JUNGE K, KLINGE U, MISEREZ M: Skin as marker for collagen type I/III ratio in abdominal wall fascia. Hernia 18: 519-525, 2014. https://doi.org/10.1007/s10029-013-1128-1

RAMANAN B, MALOLEY BJ, FITZGIBBONS RJ Jr.: Inguinal hernia: follow or repair? Adv Surg 48: 1-11, 2014. https://doi.org/10.1016/j.yasu.2014.05.017

ROSENBERG GA: Matrix metalloproteinases and their multiple roles in neurodegenerative diseases. Lancet. Neurol 8: 205-216, 2009. https://doi.org/10.1016/S1474-4422(09)70016-X

ROY R, YANG J, MOSES MA: Matrix metalloproteinases as novel biomarkers and potential therapeutic targets in human cancer. J Clin Oncol 27: 5287-5297, 2009. https://doi.org/10.1200/JCO.2009.23.5556

SMIGIELSKI J, BROCKI M, KUZDAK K, KOŁOMECKI K: Serum MMP 2 and TIMP 2 in patients with inguinal hernias. Eur J Clin Invest 41: 584-588, 2011. https://doi.org/10.1111/j.1365-2362.2010.02445.x

SMIGIELSKI J, KOŁOMECKI K, ZIEMNIAK P, DROZDA R, AMSOLIK M, KUZDAK K: Degradation of collagen by metalloproteinase 2 in patients with abdominal hernias. Eur Surg Res 42: 118-121, 2009. https://doi.org/10.1159/000187643

WANG D, HAN Y, XU X, CHEN J, CHEN Y: Matrix Metalloproteinases (MMP-2) and Tissue Inhibitors of Metalloproteinases (TIMP-2) in Patients with Inguinal Hernias. World J Surg, 2020. https://doi.org/10.1007/s00268-020-05674-0

ZÖLLER B, JI J, SUNDQUIST J, SUNDQUIST K: Shared and nonshared familial susceptibility to surgically treated inguinal hernia, femoral hernia, incisional hernia, epigastric hernia, and umbilical hernia. J Am Coll Surg 217: 289-299.e281, 2013. https://doi.org/10.1016/j.jamcollsurg.2013.04.020 